\title{
A case of complex visual disturbance in a patient with major depressive disorder successfully treated with buspar
}

\begin{abstract}
Visual perceptual disturbance, including illusions and hallucinations, can be distressing for patients with mental illness. Pareidolia is a type of complex visual illusion that occurs in health but rarely reported in patients with Depression. We present a unique case of treatment-resistant Major Depressive Disorder with co-occurring complex visual disturbance that responded to augmentation of treatment with an anxiolytic. Organic causes of visual disturbance and depression were ruled out. Additionally, toxicologic screen of biologic specimen for illicit substances was negative. A trial of treatment with an atypical antipsychotic did not improve the patient's clinical symptoms. We believe the serotonergic activity of the antipsychotic and partial agonist activity of the anxiolytic played a role in the management of this patient's perceptual and affective disturbances.
\end{abstract}

Keywords: visual perceptual disturbance, pareidolia, depression, anxiety, illicit substance, serotonergic activity, augmentation of treatment, risperidone, buspar
Volume 5 Issue I - 2018

\author{
Olawale Ojo,' Olaniyi Olayinka,' Chiedozie \\ Ojimba,' Nikhita Balabbigari, ${ }^{2}$ Nandita \\ Mahajan, ${ }^{2}$ Kang Yu,' Leon Valbrun,' Ayodeji \\ Jolayemi,' Tolulope Olupona' \\ 'Department of Psychiatry \& Behavioural Sciences, Interfaith \\ Medical Center, USA \\ ${ }^{2}$ College of Medicine,American University of Antigua, Barbuda
}

Correspondence: Olaniyi Olayinka, Department of Psychiatry \& Behavioural Sciences, Interfaith Medical Center, I545 Atlantic Avenue, Brooklyn, New York, USA, Tel I-7I 8-6I3-4334, Fax I-7I8-228-7907, Email oolayinka@interfaithmedical.com

Received: January 13, 2018 | Published: February 08, 2018
Abbreviations: CT, computed tomography; FFA, fusiform face area; MDD, major depressive disorder; MRI, magnetic resonance imaging; TBI, traumatic brain injury

\section{Introduction}

Visual perceptual disturbances in psychiatry range from absence of sensory stimuli to sensory distortion or deception. ${ }^{1}$ Although less common than auditory hallucination, the presence of visual perceptual disturbance in patients with mental illness can be distressing. ${ }^{2}$ Ascertaining the cause and type of visual perceptual disturbance occurring in symptomatic patients can be difficult to delineate and are often for descriptive purposes. ${ }^{1}$ An uncommon type of illusion is pareidolia which is a "type of complex visual illusion involving ambiguous forms being perceived as meaningful objects". ${ }^{3}$ It is a peculiar, natural, function of the human brain that makes an individual to impose patterns on random collections of images or sounds. ${ }^{3}$ The ability to recognize faces is a function of Fusiform Face Area (FFA) located in the fusiform gyrus on the temporal lobe of the human brain. ${ }^{4}$ Of note, cultural and religious backgrounds may influence visual perception. ${ }^{5,6}$ Pareidolic illusions have been reported in cases of Lewy Body Dementia and Parkinson Disease but rarely in Major Depressive Disorder (MDD). ${ }^{3}$ The presence of visual disturbances pose a treatment dilemma and often portend a worse outcome in mental disorders and neurocognitive diseases. ${ }^{2,3}$ We describe a case of Major Depressive Disorder with co-occurring vivid complex visual disturbance, and a unique approach to the management of such patient.

\section{Case presentation}

We describe the case of a 63-year-old Hispanic woman who presented to the emergency room of our hospital for evaluation of depressed mood and visual disturbances. She was agitated and distressed because of seeing the devil, and sometimes a gorilla, while looking at the faces of people. She stated that the devil/gorilla appears to be centered in a rim of moving bright colours. This distressing complex visual perception persisted with her eyes closed. On the unit, patient reported that she also saw the images when she focused on any still object, especially pictures on the wall of the unit and art work displayed in the activity room. Each experience occurred about once daily and resolved spontaneously within three hours. She also endorsed auditory hallucination of the devil and gorilla trying to talk to her and engage her during each episode of visual perceptual disturbance.

She endorsed depressed mood, anhedonia, feelings of worthlessness and poor appetite. Her depressed mood is in context of her living situation, loss of close family members and poor social support. She had only minimal interactions with peers and showed a low drive and motivation and a downcast appearance. Patient had a history of postpartum depression with a suicide attempt. Patient also had possible traumatic brain injury (TBI) from physical abuse by her maternal uncle from age 7 to 13 years. Neurological examination for pseudoseizures revealed no significant finding. Drug urine screening was negative to illicit substances. Complete blood count with differential, electrolytes, thyroid function test, and liver enzymes were normal. A computed tomography (CT) scanning of the head excluded ischemic or hemorrhagic lesions. Magnetic resonance imaging (MRI) of the brain showed a few scattered areas of hyperintensity around the ventricles and in the right frontal subcortical region on fluidattenuated inversion recovery sequence that is consistent with chronic ischemic changes/microvascular disease.

Patient was being managed on oral Risperidone and Benztropine by her outpatient psychiatrist. On the unit, she was commenced on Risperidone $2 \mathrm{mg}$ by mouth two times daily which was discontinued after two doses of Invega Sustenna maintenance was given. Symptoms remained the same with no significant improvement. She was started with Buspar $10 \mathrm{mg}$ by mouth three times daily for relief of her significant anxiety. As Buspar was titrated upward, patient showed favorable response in her mood and resolution of her illusions. 


\section{Discussion}

This case is an example of complex visual disturbance in the context of a major depressive episode which resolved following augmentation of treatment with Buspar. The cause of visual disturbance in this patient was reviewed. The complexity of the visual disturbance and the vividness of the phenomenon she described suggests pareidolia. However, it is possible that her visual hallucination is associated with a temporal lobe seizure or a serious form of synesthesia called reflex hallucination. Consideration for temporal lobe seizure was made; however, numerous neurological examination findings made the diagnosis of seizures unlikely. A history of repeated trauma to the head in childhood also raised the possibility of a TBI as some studies have reported psychosis resulting from same. ${ }^{7}$ Specifically, persecutory delusions, auditory hallucination, reduplicative paramnesias, and Capgras syndrome may occur in patients with TBI. ${ }^{7}$ On the other hand, visual perceptual disturbances are a rare long-term sequela of TBI. Additionally, neurological examination and neuroimaging did not show any focal lesions in our patient. Whether the patient's sociocultural or religious backgrounds played a role in her psychopathology is difficult to ascertain. The patient, however, was raised in western culture and admitted to being a non-practicing catholic.

Given the above, a functional cause of visual disturbance raised the suspicion of an affective etiology. There are limited studies on the link between pareidolia, MDD, and its management. Studies suggest an imbalance between serotonin and acetylcholine may lead to visual hallucination. ${ }^{8}$ Our patient received two doses of intramuscular Paliperidone following the recommended trial of oral Risperidone. Risperidone is known to have serotonergic activity. This increased activity may have contributed to our patient's visual perceptual disturbance. Regarding the mechanism by which Buspar resolved the visual misperception, it is likely that its partial agonist activity on presynaptic and postsynaptic 5-HT1 A receptors might have decreased the activity of Risperidone at serotonin receptors in the brain.

\section{Acknowledgements}

None.

\section{Conflict of interest}

The author declares no conflict of interest.

\section{Patient consent form}

Patient's informed consent to publish this case report was obtained.

\section{References}

1. Elliott B, Joyce E, Shorvon S. Delusions, illusions and hallucinations in epilepsy: 1. Elementary phenomena. Epilepsy Res. 2009;85(2-3):162171.

2. Cassano P, Chang T, Trinh NH, et al. Differential impact of isolated psychotic symptoms on treatment outcome of major depressive disorder in the STAR*D cohort of Whites, Blacks and Latinos. J Affect Disord. 2013;150(2):578-584

3. Uchiyama M, Nishio Y, Yokoi K, et al. Pareidolia in Parkinson's disease without dementia: a positron emission tomography study. Parkinsonism Relat Disord. 2015;21(6):603-609.

4. Liu J, Li J, Feng L, et al. Seeing Jesus in toast: neural and behavioural correlates of face pareidolia. Cortex. 2014;53:60-77.

5. Ishii K, Tsukasaki T, Kitayama S. Culture and visual perception: Does perceptual inference depend on culture?. Japanese Psychological Research. 2009;51(2):103-109.

6. Colzato LS, van den Wildenberg WP, Hommel B. Losing the big picture: How religion may control visual attention. PLoS One. 2008;3(11):e3679.

7. Fujii DE, Ahmed I. Psychotic disorder caused by traumatic brain injury. Psychiatr Clin North Am. 2014;37(1):113-124.

8. Lai CH. Escitalopram-related visual and auditory hallucination in a non-dementia patient with depression. J Neuropsychiatry Clin Neurosci. 2012;24(4):E19. 